\title{
Identify Occurrence of Substance Object of a Certain Classification in Fractional Videos and Pictures
}

\author{
Abhilasha Akkala, Avinash Seekoli
}

\begin{abstract}
Object detection and recognition are the meta-heuristic problems in computer vision. Practically usable dynamic object recognition methods are still unavailable. A new method was proposed which improves over existing methods in every stage. In that addition features like geometric shapes, ellipsis are added. An heuristic codebook was proposed of good generalization and discriminative properties, enabling multipath interferences mechanisms on propagation of 1 conditional livelihood. A new learning method also proposed which is capable of online learning.
\end{abstract}

Keywords : recognition, heuristic, generalization, livelihood.

\section{INTRODUCTION}

It succeeded the object detection of the goals of achieving high accuracy with a real time performance[3]. The limitation is that the dependency on other computer vision techniques for deep learning. Deep learning[1] used to solve problem object detection[4] in an end to end . It is made by the data set (Coco) which results in fast and accuracy.

\section{A. Scope}

The project aims to recognize the digits given by the users as input image. It also aims to display accuracy score of the trained model.The application strives to implement Support Vector Machine (SVC) to recognize the digits. The accuracy score is also checked.

\section{B. Objective}

Object detection[2] is increasingly been used in industries from personal security to productivity . Also used in many areas of computer vision including surveillance, image reterival.

\section{Limitations}

- Is the dimension of the output caused due to variable number of input images.

Revised Manuscript Received on February 05, 2020.

* Correspondence Author

Abhilasha Akkala*, Computer science and engineering, Centurion university of technology and management, Odisha, India. Email: a.abhilasha@cutm.ac.in

Avinash Seekoli, Computer science and engineering, Centurion university of technology and management, Odisha, India. Email: avinash.seekoli@cutm.ac.in

(C) The Authors. Published by Blue Eyes Intelligence Engineering and Sciences Publication (BEIESP). This is an open access article under the CC BY-NC-ND license (http://creativecommons.org/licenses/by-nc-nd/4.0/)
- Widespread of adsorption for real time while being accurate induction.

- Involves in classification as well as recssior.

\section{PROPOSED WORK}

Among deep learning based techniques two broad classes of methods are stage detection and unified detection

\section{A. Bouncing box}

Is the rectangular drawn on the image . Bouncing is avaible for every substance of each entity in the picture. Used to measure distance between predicted and ground truth.Shown in Fig. 1.

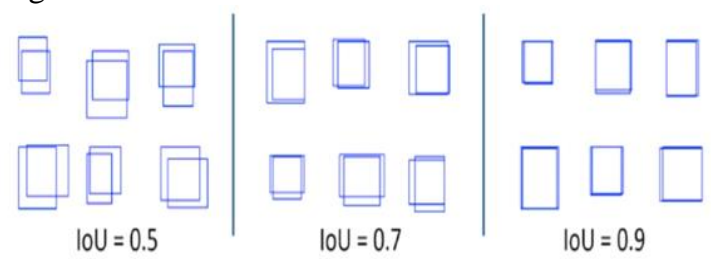

Fig-1: Jaccard distance

\section{B. Classifying Regression}

Bouncing box predicted using regression and the class within is predicted using classification.is shown in Fig. 2

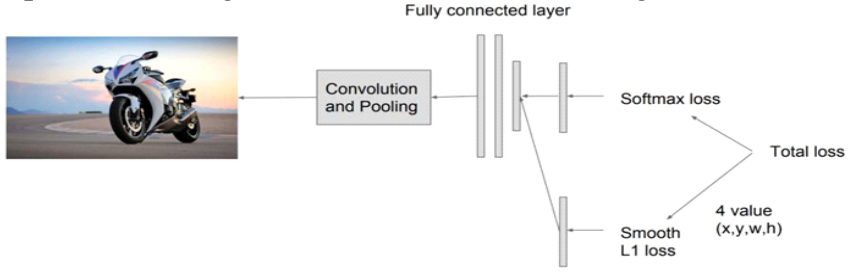

Fig-2: Architecture overview

\section{Multi-Stage process}

It is extracted and then resized to fix input for classification networks. Which acts as a feature extraction. The concept shown in Fig. 3

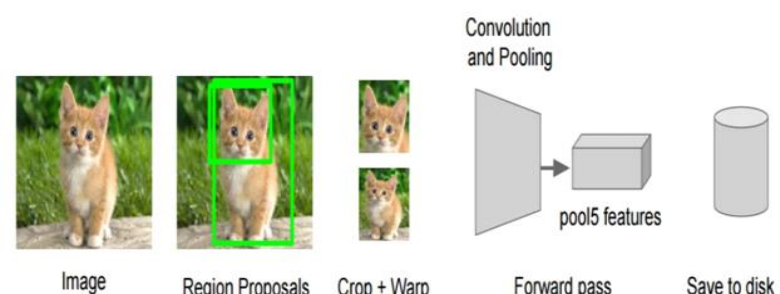

Region Proposals Crop +Warp

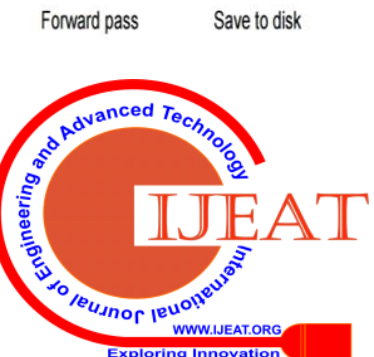




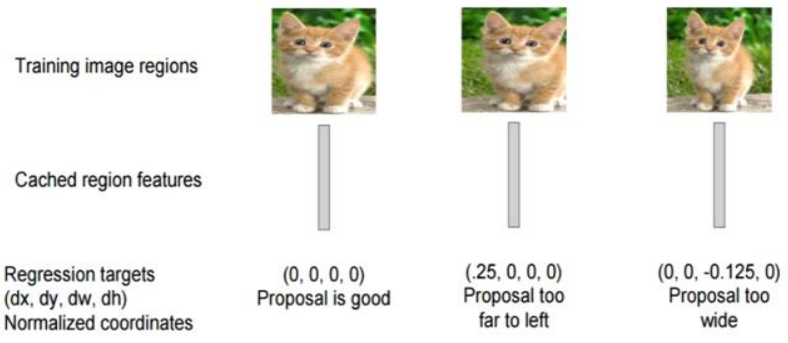

Fig-3:Multi dimension method

\section{C.Proposed method}

It is using convolutional feature maps from later layers of the network to predict class scores.

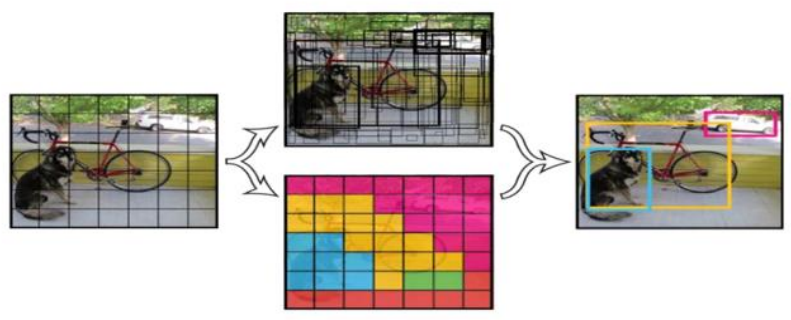

Fig-4: Proposed method

\section{YOU LOOK AT ONCE WORKING}

You look at once short name YOLO[6] has went through different iterations present YOLO9000 more feasible capable of detecting over more than 9000 object detectors,redmon and faradic are such a large number of object detection and classification. by using joint training the authors trained Yolo simultaneously on both the image net classification data set and common object detection data set. The result is shows that it can object detection but not labeled detection.yolov2's performance was a bit underwhelming given the propose work well be using yolo v3 in particular Yolo trained on the coco data set

\section{a.Common object in context}

It is excellent object detection .it consits of multiple training images and validation images.the short form of the common object is coco.many mnc are uses this data set for object detection and object segmention and so onThe COCO dataset consists of 80 labels, including, but not limited to:humans,cars, animals,some more features

- The network used in this project is based on YOLO. YOLO[4] is a very quick multi real time entity identificaion.". the entire image identification apply by neural network in this algorithm.the picture divided T X T grid by the help of network and comes up with boxes which are bounding in shape i.e the box around the images., which are boxes drawn around images and forsee the likelihood of each of these areas

-The way used to come up with the these likelihood is logit regression.Associated probabilities are used to weighted the bounded boxes. For class forsee, individual logit classifiers are used.

- By using R-CNN the areas to constrain the entity with in the given picture.the alogrithim does not capture the entire picture, it seems only only the particaular part of the pictures which have the higher chance of conaining an entity
- But present frame work identify the onject in a different approach.it will take the total picture in a one substance/instance and forsee the coordinates of bounding box

- The present algorithm is best compare to the exisisting one it is comparatively similar performance to the R-CNN algorithm for object detection

\section{B.YOLO Framework Function}

- YOLO[7] first takes an input image:

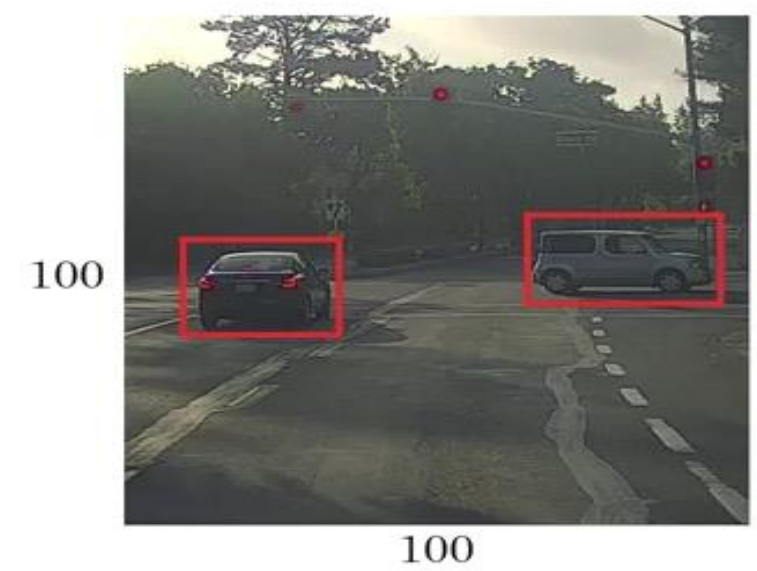

Fig-5:Input image

after the frame work is divides input image into grids

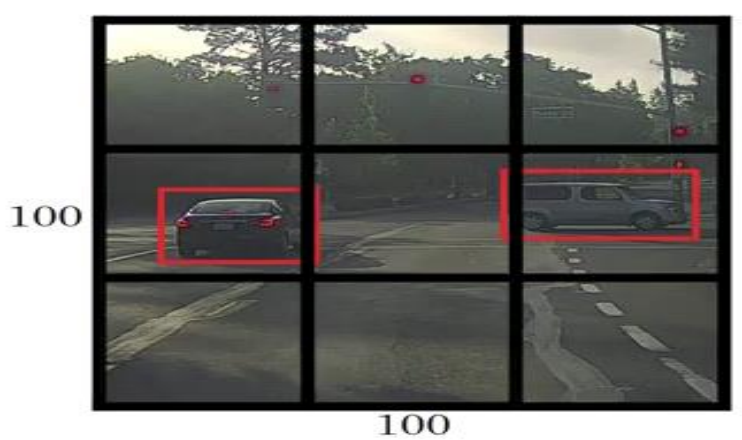

Fig-6: Grid image

- Image classifying and localisation both are applied on each phase.the forsee the bounding boxes and their match the class probabilities for entity

- for dividing the image into a grid of Size x3 . There are total 3 classes for the We need to pass the label. grid cell and the label y will be an 8-dimensional vector.

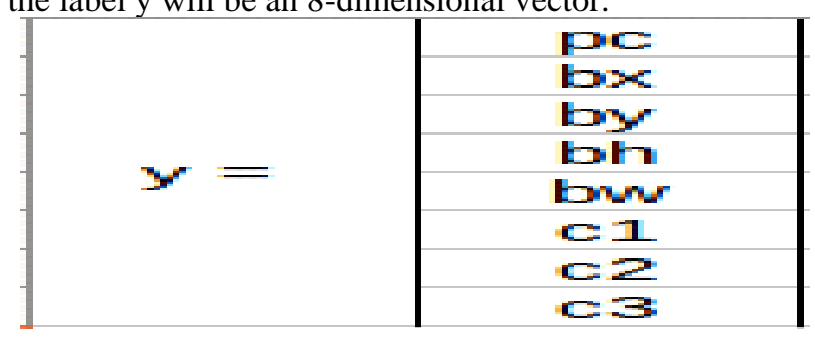

Table-I:8 dimensional data

In the aboue table p represents the entity is present in the grid or not here bx,by,bh is related to bounding box 
The notation of c1,c2,c3 represents the classes

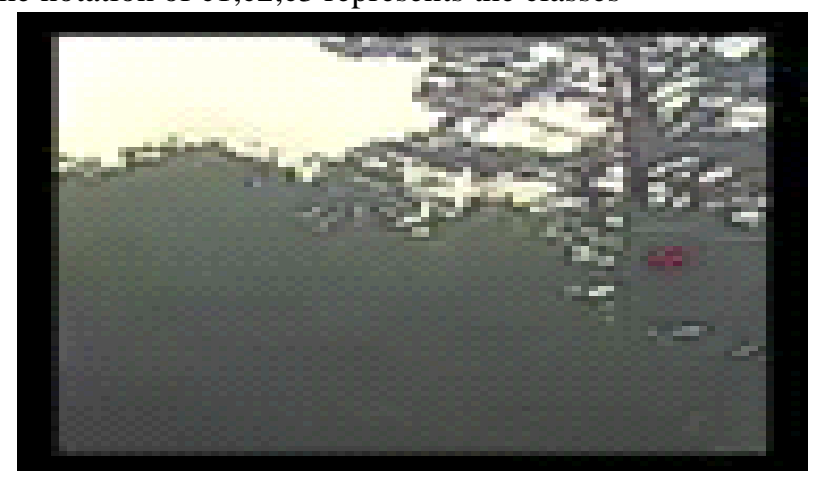

Fig-7: No object

let us sayselect the grid from the above picture

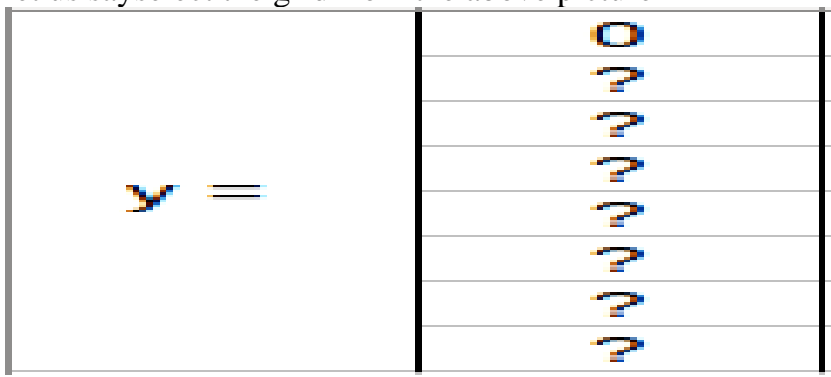

Table-II: ' 0 ' represents No object in this grid

In this picture there is no grid so the pc value is empty i.e zero then label $\mathrm{y}$ for this given picture is here the ? is not matter because there is no object in the grid so need of check classes and bounding boxes if there is no object.then go for the next condition $\mathrm{I}$,e c $2=1$

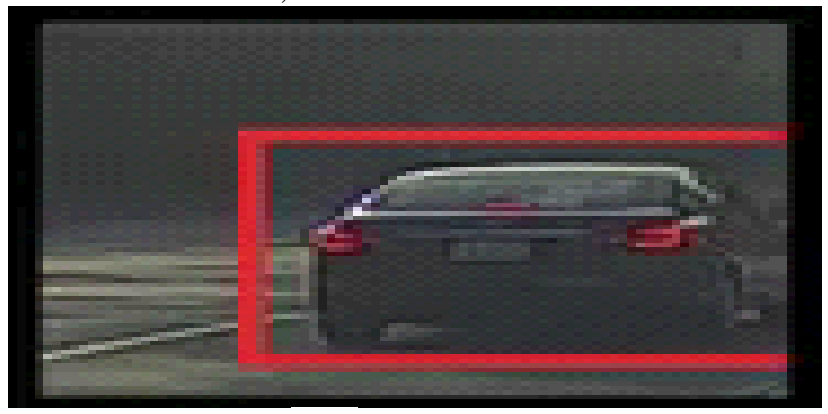

Fig-8: The center grid of the car

Here left grid with car is an entity whch is represented y With in the grid.in the above fig -8 the are multi objects so midpoint is yolo of the two objects and these entity contains mid points then these entity will assign to the grid the $\mathrm{y}$ is represent for

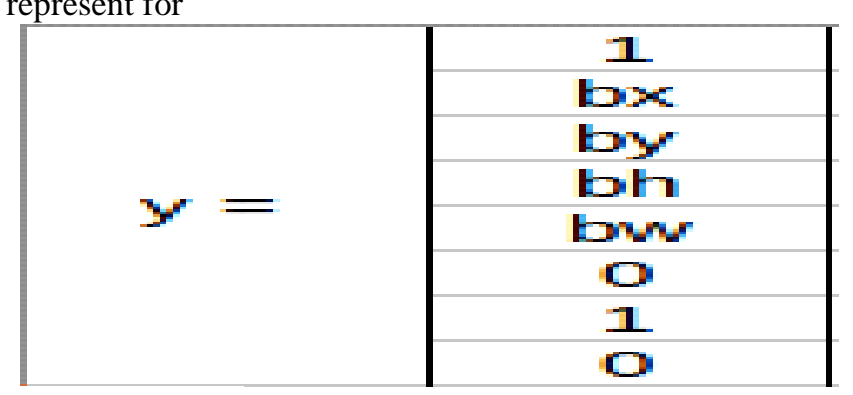

Table-III: The y represents center left grid with the car

There Is an entity in this grid ,the value of pc is equal to one ,bx,by,bh,bw will be calculated relative to the particular grid cell that we are declared,since car is $2^{\text {nd }}$ class,c2 $=1$ \& c3=0,so for 9 grids we will get an 8-dimensional output vector.the output will contain shape of $3 \times 3 \mathrm{c} 8$

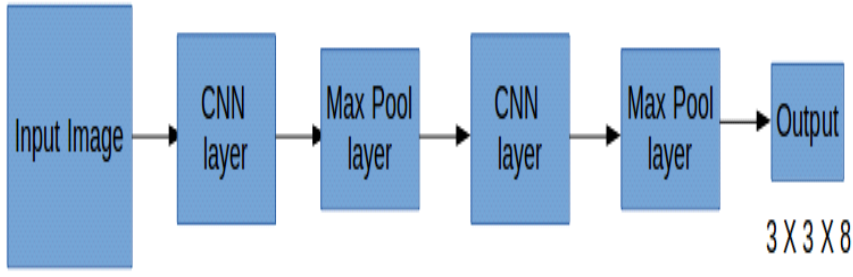

$100 \times 100 \times 3$

Fig-9: Train model

To train our model here we use forward and backward propagation to get the output $\mathrm{y}$ transfer the image to the model and apply forward propogation.but real time scenario we are taken the larger grids and also increase the number of grids due to reduce the multiple objects are appears in same grid cell

\section{CLASSIFICATION}

The image is classified into different categories one of them is image net ,recent years human performance surpassed and it has considered practically solved.

\section{A.Training data}

The new image will be of same grids will be predict an output of shape $3 * 3 * 16$. These values will be same format in training label.The first 8 values are likelihood of an entity in the 2-5 grid values will be the bouncing box cordinates for that entity belongs to.

Upto this complete the theoritical part of an algorithim , starting from training the model and generating prediction boxes for the entitie/objects

Propotions and steps of algorithim for object detection:

-takes size $(606,608,3)$ for the input image.

- transfer the picture the network (CNN) it gives the output of dimension $(19,19,5,85)$

-The last two dimensions of the above output are flattened to get an output volume $(19,19,425)$ :

- $19 * 19$ grid returns for a each cell

425 numbers.

$\bullet 425=5 * 85$, where 5 is the no of anchor boxes for grid $\bullet 85=5+80$, where 5 is (pc,bx,by,bh,bw) and 80 is the of occurence we want to identify.

- finally we do the iou and non max suppression to escape selecting overlapping boxes.

\section{A Facial identification}

Since the mid 2000 s some camera are started on efficient auto focus.It is used to detect an object in an narrower type.

\section{B. Summate}

It is very less used in object detection . in which simple count the object . Like people ,cars, flowers, and even microorganism. 
Identify Occurrence of Substance Object of a Certain Classification in Fractional Videos and Pictures

\section{C.Practical}

It also known as practical search engine of Pinterest.It works on the principle of object definition.If images are found similar which much more powerful than Google images.

\section{D.Flare picture analysis}

In the age of drone and a low budget satellites launches we are using satellite images.for many companies like planet and descreted labs . By using it we can detect any object from space in the world .(This data is very expensive before).Now we are using at in our day to day life .ex:Google map.

\section{RESULT ANALYSIS}

We used COCO dataset from DarkNet for detecting objects in our experiment. The following are the results of our experiment:

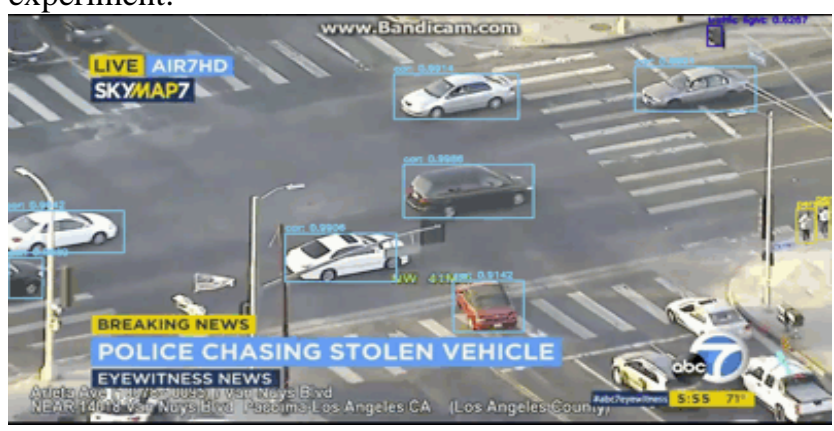

Fig-10:Police chasing stolen vehichle
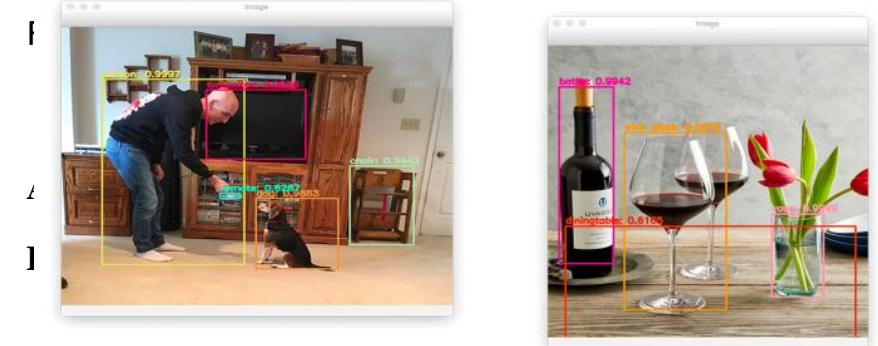

Fig-11:Data objecı uetecuuı

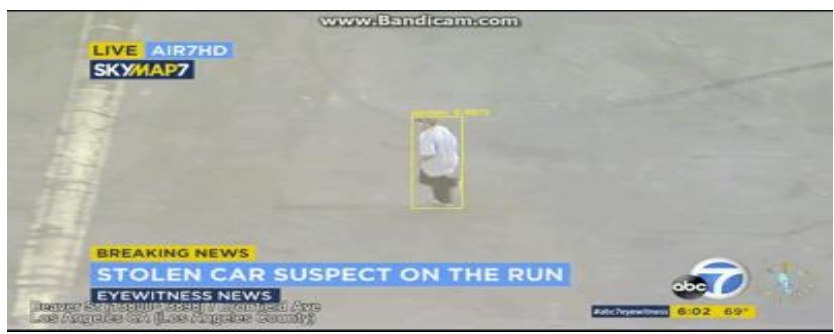

Fig-12:Stolen car suspect on the run
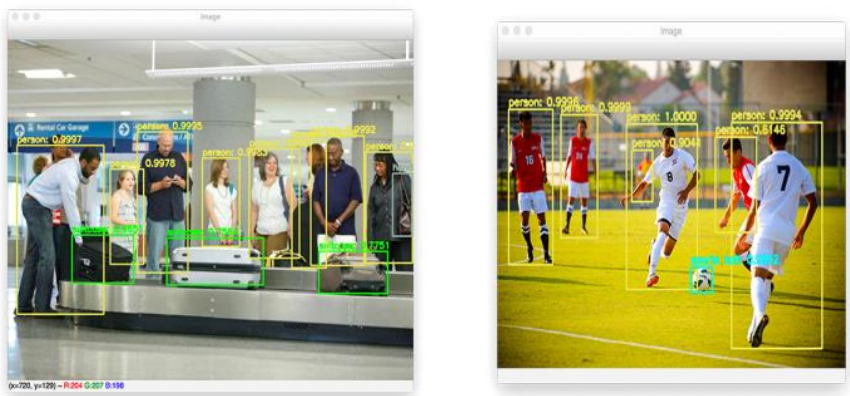

Hig-1s: עata object detection

\section{CONCLUSION}

The proposed work is more efficient compare to the previous result of existing system for object detection .here the proposed uses the techniques of deep learning in field of computer vision the data set which is created by using Yolo image and it show the evaluation is consistent.it is used for the real-time applications. To tracking the application the system is train on a video sequence.in future the algorithm will work on the accuracy for identifation objects in huge areas

\section{LIMITATIONS}

The accuracy of the trained model is $90 \%$.For each image threshold has to be changed. Can't recognize all the digits accurately.

\section{REFERENCES}

1. Michael c.burl,philip g.wetzler(2011),"Onboard object recognition for planetary exploration",Springer,34,pp-341-367

2. Ashok chandrashekar,lorenzo torresani,richard,'Learning what is where from unlabeled images:joint localization and clustering of foreground objects",springer,7,pp-261-279.

3. Paraskevi nousi et.al(2019),"embeded uav real-time visual object detection and tracking",ieee

4. Mini rani,er.Ankur gupta(2019),"recognition and detection of multiple objects from images:A review",IJARCET,8,pp-524-527

5. Minoru Asada et.al(1996),"Purposive behavior acquisition for a real robot by vision based reinforcement learning:,springer,23,pp-279-303

6. Geethapriya S,N.Duraimurgan,S.P.Chokkalingam,'Realtime object detection with YOLO",IJEAT,2019,pp-578-581

7. Gregorie Mesnil et.al(2014),"learning semantic representations of objects and their parts",springer,94,pp-281-301

8. Mahima chaddha et.al(2019), ’Deep Learning For X-Ray Image To Text Generation",IJTSRD,3,pp-1679-1682

9. Markus Schneider et.al(2016),"expected similarity estimation for large scale batch and streaming anomaly detection",springer,105,pp-305-333

10. Charalambos Eliades et.al(2018),"Automatic face recognition with well calibrated confidence measures",Springer,108,pp-511-534

\section{AUTHORS PROFILE}

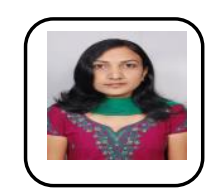

AKKALA ABHILASHA B.Tech,M.Tech(CSE),Publsih ugc journal paper,published book in Lambert Publications, Research work in Machine learning

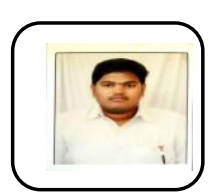

Aviinash seekoli B.Tech,M.tech(CSE), six ugc and one scopus publications,Published book at lambert publications, Membership in IJR, Research work in Software reliability, patient one registered in 2018. 